\title{
Comunicar Conocimientos vs. Organizar el Conocimiento: la Gestión del Gen-preneur en el Aula.
}

\author{
Comunicating Knowledge vs. Organising Knowledge: \\ the Use of Gen-preneur in Class.
}

\author{
Jose Manuel Comeche Martínez', Jose Vicente Pascual Ivars²
}

\begin{abstract}
Se han hecho innumerables pruebas para constatar que la creatividad es una habilidad crítica para los emprendedores y su formación emprendedora (Schmidt, J. et al. 2012), incluso se ha detectado que la práctica sobre el pensamiento divergente incrementa las habilidades emprendedoras de los estudiantes para generar un mayor número y rango de ideas, pero NO así, sus enfoques para la resolución creativa de problemas. Nuestro objetivo será mostrar que el emprendimiento es una faceta de la creatividad y que, el emprendedor "oculto" en el discente, necesitará observar y confirmar la existencia de condicionantes externos suficientes, para liberar su actitud emprendedora retenida, asimismo, será imprescindible la adopción, por parte del docente de una actitud innovadora que facilite un contexto propicio para que el alumno muestre comportamientos asociados con actitudes emprendedoras. Tras nuestro estudio, constatamos que la implementación de una actitud innovadora docente contribuye a facilitar la transmisión del espíritu emprendedor y a mejorar la gestión del gen-preneur en el aula.
\end{abstract}

Keywords: educación; comunicación del conocimiento; clase; emprendimiento; organización del conocimiento; creatividad; gen; formación.

There have been countless tests made to confirm that creativity is a critical skill for entrepreneurs and their entrepreneurial training (Schmidt, J. et al. 20I2), even more, practices on divergent thinking increases the entrepreneurial skills of students to generate a greater number and range of ideas, but not, their approaches to solve problems in a creative way. Our goal is to show that entrepreneurship is a facet of creativity and that the "hidden" entrepreneur in the learner, will need to observe and confirm the existence of sufficient external constraints -context and social network- to release its entrepreneurial attitude, furthermore it will be essential, that teachers adopt an innovative approach that enables a suitable context, in this way almost intuitively, and vocational - show student behaviors associated with entrepreneurial attitudes. In our study, we found out that the implementation of an innovative teaching contributes to facilitate the transmission of the entrepreneurial spirit and improves the use of gen-preneur in class.

Keywords: education; knowledge communication; classroom; entrepreneurship; knowledge organization; creativity; gen; training.

\footnotetext{
' Universidad de Valencia. e-mail:Jose.M.Comeche@uv.es

2 Universidad de Valencia. e-mail:Jose.pascual@uv.es
}

ISSN: 07 I8-2724. (http://www.jotmi.org) 


\section{Introducción}

Últimamente (Bocconi, S. et al. 2012; Schmidt, J. et al. 20I3; Van Treuren, K. et al., 2012) se está mostrando bastante interés en analizar las variables y condicionantes adecuados para explicar los diferentes o posibles modos adecuados para implantar el proceso innovador y de emprendimiento en el aula. Como enuncian algunos resultados (Schmidt, J. et al. 2012), los estudiantes enrolados en la clase de emprendimiento, al finalizar el entrenamiento, sí se han percibido ellos mismos como más creativos y con mejor y más pensamiento divergente; incluso se ha detectado que la práctica sobre el pensamiento divergente incrementa las habilidades emprendedoras de los estudiantes para generar un mayor número y rango de ideas, pero $\mathrm{NO}$ así sus enfoques para la resolución creativa de problemas. Esta situación plantea unos interrogantes que sería propicio analizar antes de decidir incorporar, directamente en el aula, la creatividad como canal de generación de emprendedores.

En primer lugar isería posible pensar que algunos alumnos se incorporarán a estas prácticas para evitar o disminuir el tedio que la aplicación tradicional de las clases les supone, buscando con ello una liberación de la mente y contribuyendo a la generación de mayores y mejores ideas? En segundo lugar isería posible pensar que, al haberse incorporado de forma voluntaria, los alumnos que así lo han hecho son más proclives que otros a este tipo de prácticas hacia las que, por su propio carácter tienden algún tipo de personas? En tercer lugar, ¿sería posible pensar que estamos asociando directamente creatividad con emprendimiento cuando la relación es justamente en sentido contrario?

¿Por qué se nos plantean estas preguntas? Porque la visión tradicional (Winslow \& Salomon, 1987, 1993) ha tratado de relacionar creatividad y emprendimiento viendo a la primera como una característica del segundo y suponiendo, de esta forma, que todo creativo manifiesta aspectos asociados o relacionados con el emprendimiento; de ahí la tendencia a la aplicación en el aula de técnicas que intentan estimular la creatividad como base para un incremento del factor emprendedor.

Nuestro objetivo será mostrar que la técnica de generación de mejora de habilidades cognitivas y creativas no incidirá directamente sobre el incremento del número de emprendedores, pues si bien es condición necesaria no es suficiente. El emprendedor "oculto" en el discente, necesitará observar y confirmar la existencia de condicionantes externos suficientes -contexto y red social- para liberar su actitud emprendedora retenida, asimismo, será imprescindible la adopción, por parte del docente, de una actitud innovadora que facilite un contexto propicio para que el alumno muestre comportamientos asociados con actitudes emprendedoras.
En primer lugar nos internaremos en el territorio de la creatividad, intentando dar con las claves que mejor contribuyen a transmitir y generar comportamiento emprendedor en el aula.

En segundo lugar, analizaremos el fomento de entornos facilitadores de la creatividad mediante la utilización de elementos didácticos no-formales como paso necesario para el desarrollo de una mente entrenada hacia la búsqueda de oportunidades y resolución de problemas.

$\mathrm{Y}$, en tercer lugar, intentaremos mostrar que puede existir un condicionamiento genético que predisponga a algunas personas a adoptar comportamientos que, en la escala comparativa tradicional, podrían asimilarse al comportamiento emprendedor.

\section{Claves para la transmisión del espíritu emprende- dor en el aula: definición del modelo general de creatividad}

Dada la necesidad de las empresas de generar una performance siempre cada vez más elevada, se ha procurado que la innovación y la creatividad, en la mayor parte de las organizaciones, sean vistas como una solución universal (Corrales, 1991). Sobre esta base, TRES son los elementos generalmente reconocidos en la creatividad; a saber: la regularidad, la novedad y la aceptación o el reconocimiento (Gardner, 1993; Simonton, 1999). Si observamos con detenimiento, el emprendimiento es un caso especial de creatividad. En efecto, el papel central del emprendedor es el constante -regular- deseo de "crear" algo tras la detección y reconocimiento de nuevas oportunidades. Toda la actividad del emprendedor se despliega alrededor del nacimiento de nuevas ideas (Kao, 1989). El tercer elemento determinante de la creatividad es el reconocimiento de la red social, cultural, familiar, etc., que rodea al creador y que justifica, de algún modo, la existencia de dicha creación.

Podemos entender con mayor precisión cómo el emprendimiento es una faceta particular de la creatividad y sus relaciones, a partir del modelo general de creatividad (Figura, I) definido por el autor (Comeche, 2004) y desarrollado tras el estudio detallado de lo expuesto en la literatura sobre el tema (Baronet \& Pitcher, 1995).

Las investigaciones sobre este aspecto han venido a definir $\mathrm{CINCO}$ grandes etapas o fases en el denominado proceso creador (Figura 2). Aquí presentamos lo que pudiera ser una descripción aproximada de lo que pasa en el cerebro y el corazón del individuo creador. Los creadores construyen 
un plano mental, entendido como una "organización personal" de conocimientos que representa, probablemente, el elemento cognitivo más importante en la creatividad. De hecho, es esta "organización" de conocimientos lo que le permitiría el necesario pensamiento divergente asociado a la originalidad y a la innovación. Vislumbramos aquí una de las claves de transmisión del espíritu emprendedor o como se enuncia en el título, de la gestión del gen-preneur en el aula, al menos, de forma tangencial a lo que se analizará en profundidad, más adelante: no se puede enseñar a emprender, debemos enseñar a "organizar" conocimientos para potenciar un mayor rango de pensamiento o conocimiento divergente en el discente para, por ejemplo, detectar nuevas oportunidades, nuevas carencias o necesidades o deseos para hacer nuevas cosas -creaciones/emprendimientos- y acercarlas al flujo mercantil, es decir, innovar.

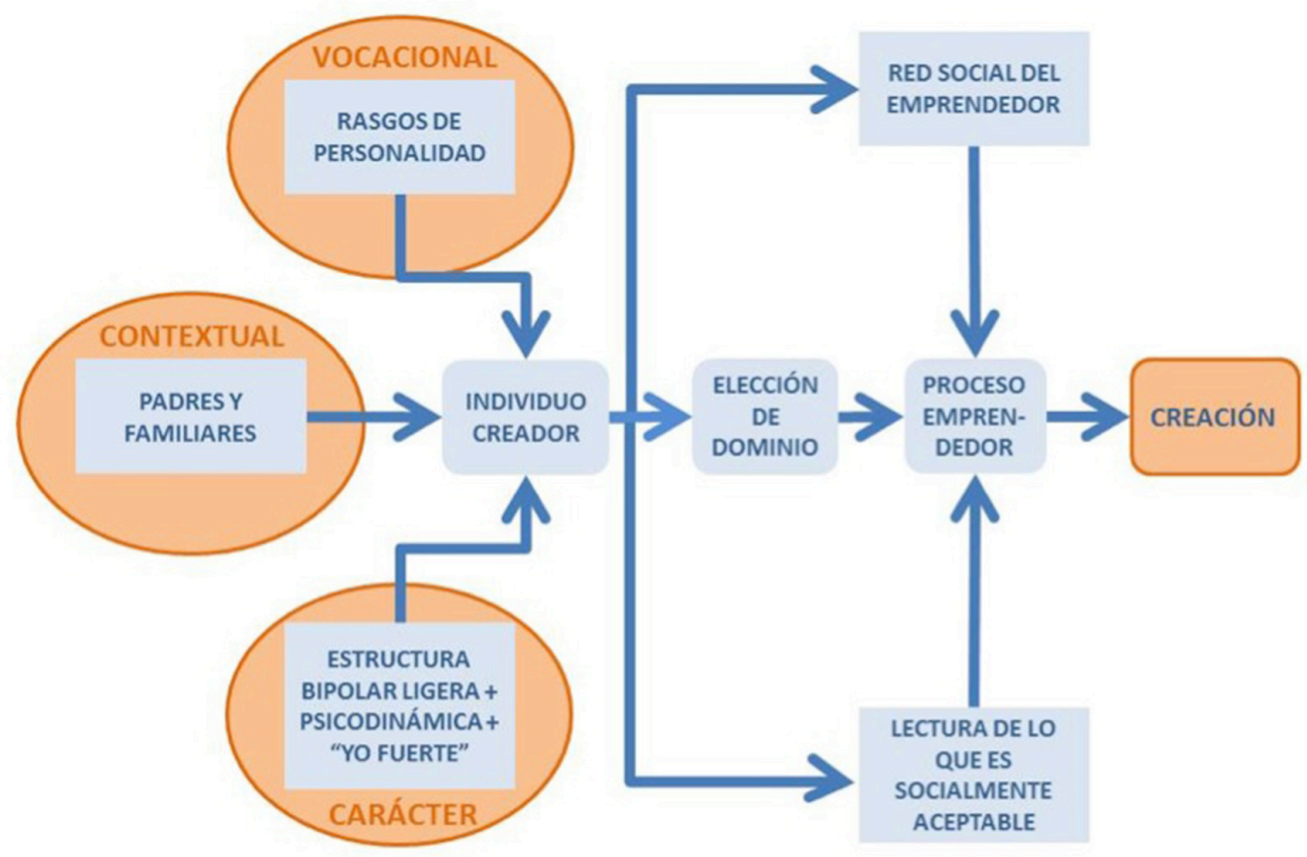

Figura I.- Modelo general de creatividad. Fuente:Adaptado de Baronet y Pitcher (1995).

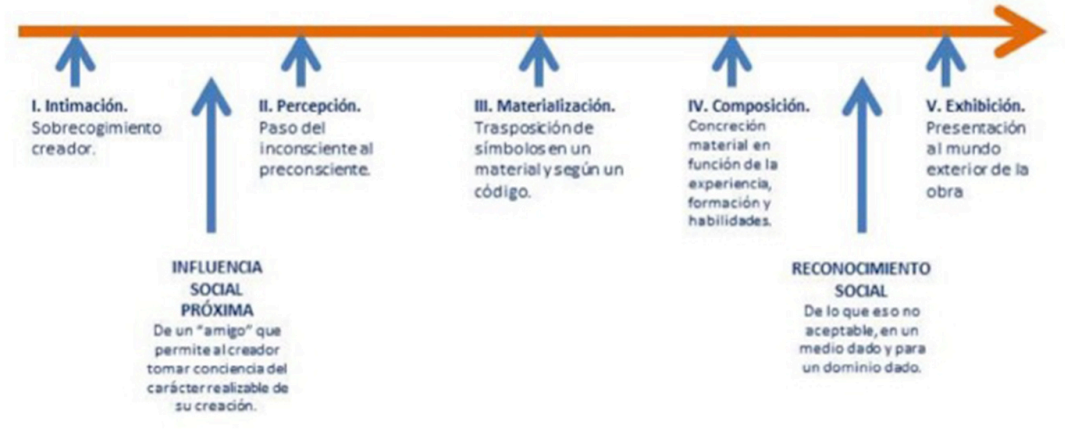

Figura 2.- Modelo del proceso creador. Fases. Funte: Elaboración propia.

ISSN: 07 I8-2724. (http://www.jotmi.org)

Journal of Technology Management \& Innovation (c) Universidad Alberto Hurtado, Facultad de Economía y Negocios. 
Durante la primera etapa el individuo creador está literalmente "embargado/recogido" como en un "episodio psicótico —aunque no patológico- temporal" (Anzieu, 1974). Se habla aquí de una excitación confusa, de un vivo deseo y de una clase de "intimación" a perseguir cualquier cosa fundamental (Eysenck, 1994). Los psicoanalistas lo denominan una "regresión al servicio del yo" (Suler, 1980; Anzieu, 198I), la cual crea una fuerte perturbación. De ahí la necesidad de un "yo fuerte" en el carácter del individuo creador (Simonton, 1999). La segunda fase del proceso creador es una fase de percepción, de paso del inconsciente al preconsciente del individuo creador, normalmente gracias a terceras personas que hacen apreciar al creador la factibilidad de su deseo creador. En la tercera fase, el individuo creador materializa su creación, según los códigos del dominio de su actividad, es decir, en su campo de experiencia.

La cuarta fase es la correspondiente a la "composición" propiamente dicha. Es donde el individuo creador utiliza su conocimiento de la actividad donde él trabaja, su experiencia, su formación formal e informal y sus habilidades para concretar lo que ha sentido y decodificado. En esta fase y en la siguiente, el reconocimiento de lo que es aceptable, en un medio dado y por un dominio dado, entra en juego. Por tanto, para el individuo creador representa un inicio de compromiso entre lo que él desearía verdaderamente expresar y lo que él debe hacer "para que esto pase". La quinta y última fase del proceso creador es la de la exposición de la obra creada al mundo exterior. Algunos creadores no han pasado de la cuarta fase y no han asumido los compromisos necesarios entre sus emociones y conflictos, y lo que es aceptable en el entorno, conservando unas creaciones que no han sido conocidas por el público y que, por consiguiente, quedan al nivel de un sueño. En el mundo de los negocios en general y del emprendimiento en particular, por ejemplo, esta fase hace referencia a la puesta en funcionamiento en el mercado de una nueva idea o de un nuevo producto (Land, 1975).
No obstante lo anterior, el individuo emprendedor forma parte de un campo, de una red de personas e instituciones que juzgan su trabajo, que le ayudan y que, en ocasiones, incluso, entran en competición con él (co-creación). En los trabajos de Moore (1986) y Bygrave (1989) se pone de manifiesto la influencia de las redes formadas por stakeholders conjugando las características psicológicas del emprendedor $y$ el nivel del entorno activo de la puesta en escena de la "nueva empresa" (Bygrave \& Hofer, 199l). La red ayuda, igualmente, al individuo creador a salir de su aislamiento natural (Ripollés y Menguzzato, 2004), construyendo, junto con los distintos agentes de su entorno económico y social una importante fuente de recursos para llevar a cabo su creación o empresa (lborra, et al., 1998; Ripollés et al., 2002). Aparece aquí una nueva clave, es la relativa a la necesidad de capacitar al individuo -alumno- en el desarrollo de habilidades sociales que le permitan interactuar eficientemente con la red social que define su entorno cognitivo, para lo cual se deberán incorporar técnicas que le permitan comunicar eficazmente sus emociones e intenciones internas. Para poder estimular el desarrollo de habilidades sociales que le permitan al creador, potencial emprendedor, interactuar eficientemente con la red social que le envuelve debemos, primero, identificar y definir las capacidades constitutivas del talento creativo, tales son: la fluidez, la flexibilidad y la originalidad (Durán, C., 2006).Además, el talento creativo incluye otras características que no son estructurales; como son: la tendencia a la redefinición, la elaboración, la sensibilidad ante los problemas y la evaluación (Herrán, A., 2008); las personas genuinamente emprendedoras se destacan, además, por una potente motivación de obra, esto es, por la perseverancia en los proyectos creativos, para asumirlos como aspectos esenciales de los proyectos de vida pues constituyen su life motive interior.

\section{Generación de entornos favorables en el aula: me- todología y didáctica estimulativas no formales.}

El contexto del creador es determinante en el factor creativo aplicado al aula.A este respecto, antropólogos e historiadores han identificado algunos factores del ambiente físico relacionados con el auge cultural. La interacción de factores atmosféricos, geográficos y biofísicos como el clima, pueden propiciar condiciones favorables para la inventiva pues, disponer de tiempo libre permite cultivar la creatividad. Pero, de otro lado, un ambiente excesivamente confortable podría ser contrario a la invención, pues no permitiría el enfrentamiento de condiciones adversas, verdadero motor de la inventiva (Correa, P., 20II). 
Veamos pues algunas de las condiciones fundamentales concebidas como una forma de mejora contextual en el aula que influyen en el ambiente creativo, como algunos autores señalan, para el desarrollo de la creatividad en la educación:

a) En primer término, una clara predisposición por parte del profesor (Torrance \& Myers, 1976); lo que equivale a decir que inicialmente, no todo el profesorado posee las actitudes $y$ habilidades necesarias para ofrecer paradigmas válidos en este campo.

b) El profesor no ha de reprimir las respuestas originales ni enjuiciar de manera restrictiva, aunque se razone sobre su viabilidad (Fernández Huerta, 1968).

c) La creatividad educativa supone incorporar la incertidumbre entre los objetivos a conseguir (Seiber, 1978).

d) Fomentar las situaciones de contraste y destacar el papel que juega la ironía y el humor (Marín, 1980).

e) Otras veces las asociaciones creativas surgen por la proximidad espacio-temporal -choque de culturas- (Marín, 1980).

f) El profesor creativo recurre a la anticipación de respuestas y su simulación, como ejercicios fomentadores de la inventiva (Sandi, 1980).

g) Por último, no podemos olvidar que, aplicada al terreno científico, creatividad equivale a investigación.

Si trasladamos la problemática al aula y recordamos que la creatividad es un tipo de aprendizaje eminentemente sintético, el primer principio didáctico que aparece como de necesaria aplicación será la necesidad de diversificar las fuentes informativas y formativas (Sarramona, 1984). Efectivamente, si se diversifican los textos informativos, ineludiblemente se hará necesaria la síntesis personal para armonizar y estructurar los conocimientos adquiridos.

Sobre esta base, podemos enunciar otro segundo grupo de principios didácticos de aplicación en el aula (Maddison, 197I):

- La utilización de recursos audiovisuales para fomentar la creatividad verbal.

- La dramatización no visual -radio-, donde cada oyente genera un espacio temporal diferente al recrear lo oído (Murga, 1983; Pastor y Xifra, 2010).

Y, por último, no podemos dejar de resaltar la evidencia que la enseñanza por ordenador representa en el ámbito de la metodología no-formal en su sentido más pleno. Efectivamente, estrechamente ligada con la resolución de problemas, está la aportación del ordenador en la creación de simulaciones, que, como ha quedado más que expuesto, constituye una de las técnicas válidas para despertar el pensamiento creativo (Imbernón, Silva y Guzmán, 20I I). El camino, pues, parece claro: los medios de comunicación han de penetrar, aún más si cabe, en los programas de educación de adultos y allí ser usados en beneficio de metas optimizadoras, entre las cuales la creatividad no puede faltar. Permitiendo generar, estimular y desarrollar en el estudiante habilidades que le permitan interactuar eficientemente con la red social que define su entorno cognitivo e incorporando técnicas que le permitan comunicar eficazmente sus emociones e intenciones creativas y emprendedoras.

\section{La perspectiva genética y el emprendimiento: un factor más a tener en cuenta.}

Tenemos la convicción de que se puede enseñar a poner en marcha un negocio, se puede ayudar a ser más creativo, pero no se puede enseñar a ser emprendedor, porque "...se les puede enseñar técnicas y prácticas específicas, pero no se les puede enseñar en el famoso "will to conquer"' (Falck, et al., 20/2). Efectivamente, este atributo del emprendedor es parte esencial de la identidad de la persona. Llegados a este punto, podríamos preguntarnos ¿Se nace emprendedor? ¿Es posible que algunos genes puedan hacer a algunas personas más sensibles que otras a los estímulos ambientales? ¿Existe una genética que predispone a la persona hacia comportamientos emprendedores? Creemos que puede haber una clara conexión entre la predisposición y el comportamiento emprendedor y ciertos condicionantes genéticos, a los que igualmente, para no ser tan estrictos, podríamos llamar, vocacionales.Y esa sería una respuesta al por qué, de que quienes se lanzan como voluntarios a participar en los ensayos sobre Creatividad vs. Emprendimiento muestran, al finalizar, actitudes y aptitudes un tanto más desarrolladas que quienes participan en el grupo de control. Probablemente, están más predispuestos genéticamente que los otros o al menos así se perciben ellos mismos (Nicolaou \& Shane, 2009).

Durante 40 años los investigadores han examinado quién y por qué se convierte en emprendedor (Gartner, 1988; Bird, 1989; Shane \& Venkataraman, 2000; Aldrich \& Martinez, 200I; Baron, 2004). Sin embargo, una de las más interesantes explicaciones de la tendencia de las personas a iniciar una actividad emprendedora no ha sido ampliamente examinada (la perspectiva genética o "vocacional"). Diversos estudios (Nicolaou \& Shane, 2009) advierten, que los factores genéticos pueden influir sobre la tendencia de la personas a participar en la actividad emprendedora. Tal es el efecto del denominado factor geno-ambiental en la infancia que, es más que aceptado que la identidad empresarial ${ }^{2}$ junto con

\footnotetext{
'Voluntad de conquistar.

${ }^{2}$ Entrepreneurship Identity = Identidad empresarial

(Falck, et al., 20I2)
} 
determinadas habilidades, resulta de una socialización del individuo en el hogar y en la escuela (Akerlof \& Kranton, 2005). Parece plausible asumir, por tanto, que una identidad inclinada al emprendimiento empresarial se desarrollará de forma similar, es decir, construyendo entornos (escuela y familia) que favorezcan el desarrollo de capacidades cognitivas emprendedoras. Por otro lado, sabemos que los genes direccionan a las personas a seleccionar, modificar y construir su entorno (Scarr, 1992), y que los factores medioambientales no son distribuidos al azar entre las personas de diferente composición genética (Neale \& Maes, 2002). Por ello, los genes también pueden influir en la tendencia de algunas personas a iniciar o participar en actividades emprendedoras a través de la selección de otras personas (socios) en diferentes entornos.

Un largo número de estudios han proporcionado evidencias consistentes de la relación positiva entre la educación y la actividad emprendedora, tanto en EEUU como en Suecia, el Reino Unido y Finlandia (Borjas, 1986; Rees \& Shah, 1986; Macpherson, 1988; Borjas \& Bronars, 1989; Boyd, 1990; Fernández \& Kim, 1998; Delmar \& Davidsson, 2000; Ritsila \& Tervo, 2002; Davidsson \& Honig, 2003). Hallando pues evidencia empírica de que la educación está afectada por factores genéticos, parece lógico pensar que los factores genéticos influyen en el nivel de educación que la gente obtiene. Dado, por tanto, que las personas con más alto nivel educativo son más proclives que otras a iniciar o participar en actividades emprendedoras, podríamos pensar que las interacciones geno-ambientales influyen no solo en la tendencia de algunas personas a iniciar o participar en actividades emprendedoras, más aún, influyen incluso a la hora de seleccionar -de manera, a veces intuitiva-, compañeros de viaje (colegio, instituto, vecindad, club, empresa, despacho, etc.) pues en algunos estudios (Falck, O., et al., 2012) se ha encontrado soporte empírico suficiente a la hipótesis de que "el hecho de tener compañeros emprendedores en la escuela -etapa escolar- tiene un elevado impacto en el desarrollo de las intenciones emprendedoras/empresariales futuras del individuo, en ese grupo de edad (alrededor de los 15 años).Además, de encontrarse suficiente evidencia de que las intenciones empresariales en la adolescencia son un claro y buen predictor para convertirse en un futuro empresario". Lo expresado anteriormente nos seduce de tal manera que nos incita a considerar el factor geno-ambiental como determinante a la hora de identificar e, incluso, de generar entornos adecuados para que nuestro alumno, futuro emprendedor, encuentre las condiciones necesarias y suficientes para explosionar todo su potencial interior.

\section{Estudio empírico}

Para contrastar lo expresado anteriormente, los autores pusieron en marcha, en los últimos tres cursos (2009_12), un proyecto de innovación educativa en el aula, tendente a estudiar cómo la variación de algunos aspectos contextuales, metodológicos y didácticos, podían influir sobre la percepción respecto de la capacidad creativa y la orientación emprendedora, que sobre sí mismos tienen los alumnos y cómo éstas podían evolucionar a lo largo del proceso. El estudio se realizó sobre un grupo de alumnos de la Diplomatura de Turismo que, en años sucesivos, voluntariamente accedieron a ser analizados. Dado que algunos alumnos fueron quedando desplazados del grupo, el número de alumnos que finalmente fueron objeto de estudio es de 23 , número correspondiente a los alumnos que han permanecido en el Proyecto los citados tres cursos.

La ficha técnica correspondiente a la muestra utilizada se define a continuación (tabla I).

\begin{tabular}{|l|l|l|l|l|}
\hline Curso & $\mathbf{N}^{\circ}$ alumos & Asignatura & Procesos & $\begin{array}{l}\text { Innovación } \\
\text { docente* }\end{array}$ \\
\hline 2009_10 & 29 & Org.y Gest. de Empresas: Los RH & Fase I & $\begin{array}{l}\text { Cambio en la actitud } \\
\text { del profesorado: } \\
\text { visión innovadora, }\end{array}$ \\
\hline 2010_II & 25 & Direcc. Estrat. de Empresas Turísticas & Fase II & $\begin{array}{l}\text { Generación de En- } \\
\text { tornos favorables }\end{array}$ \\
\hline 2011 I2 & 23 & Creac. Empresas Turísitcas & Fase III & $\begin{array}{l}\text { Técnicas, recursos y } \\
\text { herramientas didác- } \\
\text { ticas. }\end{array}$ \\
\hline
\end{tabular}

Tabla I: Ficha Técnica muestal. 


\section{Metodología}

Nuestro estudio comenzó en los años 2009_10, sobre un grupo de alumnos de la Diplomatura de Turismo orientado hacia la Creación de Empresas, concretamente en la asignatura de Organización y Gestión de Empresas: los RRHH. Tras la necesaria explicación de los objetivos, perspectivas y alcance de nuestra investigación, y antes de comenzar a impartir las clases propias del contenido de la asignatura, se procedió al pase de un cuestionario diseñado al efecto, para obtener información respecto de la percepción que sobre la vertiente emprendedora tenía de sí mismo el alumno/a y cuál era la valoración de su perfil emprendedor. Esta información nos iba a servir de referencia para contrastar una posible evolución tras la implementación de los procesos de innovación docente diseñados para intentar mejorar la orientación creativa y emprendedora del alumno.

\section{Actuaciones}

Nuestra primera intención consistió en alterar la metodología tradicional docente implementando, en este primer año, una visión diferente e innovadora, es decir, aplicar las denominadas claves en la transmisión del espíritu emprendedor para una mejor gestión del gen-preneur en el aula; a saber, la generación de los denominados "grupos de emprendimiento", junto con la necesaria capacitación para organizar conocimientos y no la pura comunicación de información, así como una dedicación especial y personalizada a cada alumno para, en función de su personalidad, ayudarle a desarrollar su potencialidad social y de comunicación.

En el curso 2010_I I, en la asignatura Dirección Estratégica de Empresas Turísticas, impartida en el segundo curso de la Diplomatura de Turismo, y sobre un grupo de alumnos que constituía más de un $85 \%$ de los alumnos ya analizados, se inició la Fase II, en la que además de las prácticas anteriores se procedió a incorporar una nueva innovación docente. Tal consistió en alterar el comportamiento del profesorado hacia la generación de un ambiente propicio para el desarrollo de la creatividad en la educación, como ya se ha explicado. La utilización de prácticas docentes basadas en la aceptación de la ambigüedad, el fomento del contraste, el humor y la ironía y una clara predisposición del profesor hacia la estimulación ideática no restrictiva, suponíamos que afectaría sustancialmente a la valoración que sobre el perfil emprendedor hacían de sí mismos los alumnos, como muestran los resultados que más tarde analizamos.

En el último de los cursos objeto de estudio, 20II_12, Fase III, se analizaron las posibles implicaciones que; sobre un grupo de 23 alumnos, que permanecen en el estudio, de la asignatura Creación de empresas Turísticas perteneciente al tercer curso de la Diplomatura y con un conjunto común de alumnos superior al $90 \%$ de los analizados en el curso anterior, podía tener la adición de un tercer bloque de medidas de innovación docente en la gestión del gen-preneur en el aula; a saber, la puesta en marcha de los denominados principios didácticos consistente en la utilización de técnicas, recursos o herramientas que ayudan a mejorar el aprendizaje, tales como la diversificación de las fuentes de formación e información, la utilización de recursos audiovisuales basados en técnicas de dramatización no visual, etc., etc.

En base a lo anterior, podemos presentar las siguientes hipótesis:

Hipótesis I.- La implementación en el aula de innovaciones docentes consistentes en la generación de los denominados "grupos de emprendimiento", junto con la necesaria capacitación para organizar conocimientos y la utilización de técnicas para mejorar su potencialidad social y de comunicación; influirán positivamente en la percepción de la orientación creativa y emprendedora que de sí mismos tienen los alumnos.

Hipótesis 2.- La implementación en el aula de innovaciones docentes consistentes en la utilización de prácticas docentes basadas en la aceptación de la ambigüedad, el fomento del contraste, el humor y la ironía y una clara predisposición del profesor hacia la estimulación ideática no restrictiva; influirán positivamente en la percepción de la orientación creativa y emprendedora que de sí mismos tienen los alumnos.

Hipótesis 3.- La implementación en el aula de innovaciones docentes consistentes en la utilización de técnicas, recursos o herramientas que ayudan a mejorar el aprendizaje, tales como la diversificación de las fuentes de formación e información, la utilización de recursos audiovisuales basados en técnicas de dramatización no visual, etc., etc.; influirán positivamente en la percepción de la orientación creativa y emprendedora que de sí mismos tienen los alumnos.

Tales hipótesis se intentaron contrastar a través del análisis de la diferencia de medias aportado por la aplicación de la " $t$ " de Student sobre el colectivo muestral definido.

\section{Resultados}

Tras el primer cuatrimestre de experimentación (Fase I), y junto a la constatación vivencial por parte del profesor de una mejora sustancial en las actitudes y posicionamiento emprendedor del conjunto de la clase, analizamos los resultados obtenidos del estudio del cuestionario. En la Tabla 2 podemos comprobar la diferencia existente entre la percepción que sobre el perfil emprendedor tienen los alumnos a principio de curso y al final del mismo. Vemos como se produce un aumento de la " $t$ ", ante diferencias de medias 
significativas, lo cual nos lleva a concluir que ha mejorado el perfil emprendedor del alumnado, dado que el valor de " $t$ " es superior al valor de la " $t$ " crítica para 22 grados de libertad y una probabilidad del $0,01 \quad(t=2,5)$. Lo cual nos lleva a confirmar la Hipótesis I de partida.

El segundo curso de experimentación contribuyó a aportar al informe del profesor una mejora sustancial en la percepción que de las contribuciones, comportamientos y capacidades se tuvieron el curso anterior. No obstante, el análisis de resultados del cuestionario sobre perfil emprendedor se presenta a continuación. En la Tabla 3 podemos observar los resultados obtenidos tras el análisis de diferencias de medias realizado. Los resultados confirman que existe una diferencia de medias significativa y que el valor de la " $t$ " aumenta para un mismo valor de " $t$ " crítica, respecto del valor obtenido el curso anterior, con un nivel de significatividad superior al $99 \%$. Estos resultados confirman nuestra Hipótesis 2, respecto de que al aplicar la segunda innovación docente, la percepción que sobre el perfil emprendedor tiene el propio alumnado participante mejora significativamente.

Para el análisis de los resultados de aplicación de la Fase III, procedimos de la misma forma que en las fases anteriores. Como se puede apreciar por el análisis de los resultados, la tendencia evolutiva parcial sigue siendo positiva y la evolución global del grupo de análisis es más que satisfactoria; lo cual nos confirma nuestra Hipótesis 3 de partida. En la Tabla 4 podemos observar dichos resultados obtenidos de nuevo tras el análisis de la diferencia de medias.

Tras haber confirmado las Hipótesis planteadas y aplicando una visión global a nuestro estudio, procedimos a analizar los resultados comparando el perfil emprendedor percibido antes de implementar las innovaciones docentes anteriormente reseñadas y tras la implementación de los tres bloques de innovaciones (final del tercer período). Los resultados se muestran en la Tabla 5 a continuación.

\begin{tabular}{|l|l|l|l|l|}
\hline Primer Período & $\mathrm{N}$ & Media & Desviación típ. & $\mathrm{t}$ \\
\hline Perfil Emprendedor (2009-10)(1) & 23 &, 4348 &, 50687 & $4,114^{\star \star *}$ \\
\hline Perfil Emprendedor (2009-10)(2) & 23 &, 5652 &, 50687 & $5,348^{\star \star *}$ \\
\hline
\end{tabular}

Tabla 2: Diferencia de medias ler curso primer y segundo período *** Estadísticamente significativo a un nivel superior al 99\%. T Crítica $=2,5$

\begin{tabular}{|l|l|l|l|l|}
\hline Segundo Periodo & $\mathrm{N}$ & Media & Desviación típ. & $\mathrm{t}$ \\
\hline Perfil Emprendedor (2009-10)(2) & 23 &, 5652 &, 50687 & $5,348^{\star \star \star}$ \\
\hline Perfil Emprendedor (2010-11) & 23 &, 6957 &, 47047 & $7,091^{\star \star *}$ \\
\hline
\end{tabular}

Tabla 3: Diferencia de medias tras el segundo período *** Estadísticamente significativo a un nivel superior al 99\%. T Crítica= 2,5

\begin{tabular}{|l|l|l|l|l|}
\hline Tercer Período & $\mathrm{N}$ & Media & Desviación típ. & $\mathrm{t}$ \\
\hline Perfil Emprendedor (2010-11) & 23 &, 6957 &, 47047 & $7,091^{\text {*** }}$ \\
\hline Perfil Emprendedor (2011-12) & 23 &, 7391 &, 44898 & $7,895^{\text {}}$ \\
\hline
\end{tabular}

Tabla 4: Diferencia de medias tras el tercer período *** Estadísticamente significativo a un nivel superior al 99\%. $\mathrm{T}$ Crítica= 2,5

\begin{tabular}{|l|l|l|l|l|}
\hline Tercer Periodo & $\mathrm{N}$ & Media & Desviación típ. & $\mathrm{t}$ \\
\hline Perfil Emprendedor (2009-10)(1) & 23 &, 4348 &, 50687 & $4,114^{\star \star *}$ \\
\hline Perfil Emprendedor (2011-12) & 23 &, 7391 &, 44898 & $7,895^{\star \star \star}$ \\
\hline
\end{tabular}

Tabla 5: Diferencia de medias entre inicio y final del proceso *** Estadísticamente significativo a un nivel superior al 99\%.T Crítica= 2,5

ISSN: 07 I8-2724. (http://www.jotmi.org) 


\section{Conclusiones y vías de investigación futura}

Tras nuestro estudio, podemos constatar que, efectivamente, la implementación y puesta en acción de una actitud innovadora docente a través de las denominadas "claves" contribuye a facilitar la transmisión del espíritu emprendedor y a mejorar la gestión del gen-preneur en el aula.

La primera de ellas reside en el comportamiento del profesorado, que debería ser orientado hacia la generación de entornos favorables. En segundo lugar, mantener una actitud dinamizadora hacia el alumno que sea proclive a la generación de grupos de trabajo. Lo cual nos permitirá generar los denominados "grupos de emprendimiento" sobre los que nos será fácil aplicar las demás claves para generar y transmitir $y$, por qué no, desarrollar el espíritu emprendedor en el aula.

Otra de las claves que debemos tener en cuenta, consiste en el cambio de actitud, por parte del profesorado, respecto de la tendencia de éste a enseñar a emprender como prácticamente el único camino hacia el emprendimiento. Insistimos, debemos enseñar a "organizar" conocimientos como herramienta para potenciar en el alumno la rutina del pensamiento divergente orientado hacia la detección de oportunidades y la innovación.
La tercera clave sería la relativa a la necesidad de capacitarle en el desarrollo de habilidades sociales que le permitan interactuar eficientemente con su red social; incorporando, para ello, técnicas de comunicación eficaz que le permitan transmitir sus emociones, sus intenciones y sus proyectos, $y$ todo aquello que fluye desde su interior emprendedor.

Otra vertiente a considerar, es la perspectiva vocacional del estudiante, los entornos vivenciales de la infancia y la juventud, la familia, los compañeros de viaje, etc... son determinantes a la hora de potenciar el espíritu emprendedor, por ello, es vital la construcción de entornos (familia, amigos, escuela...) que favorezcan el desarrollo de las capacidades cognitivas y emprendedoras del alumno.

Estos principios, aplicados en el aula, nos permiten diseñar un modelo de gestión del factor gen-preneur (Figura 3) de tal forma que podemos mejorar, estimular y desarrollar en el estudiante toda una serie de habilidades que le facultarán para interactuar más eficientemente con la red social que define su entorno cognitivo; comunicando de forma más eficiente sus intenciones, emociones e intuiciones emprendedoras.

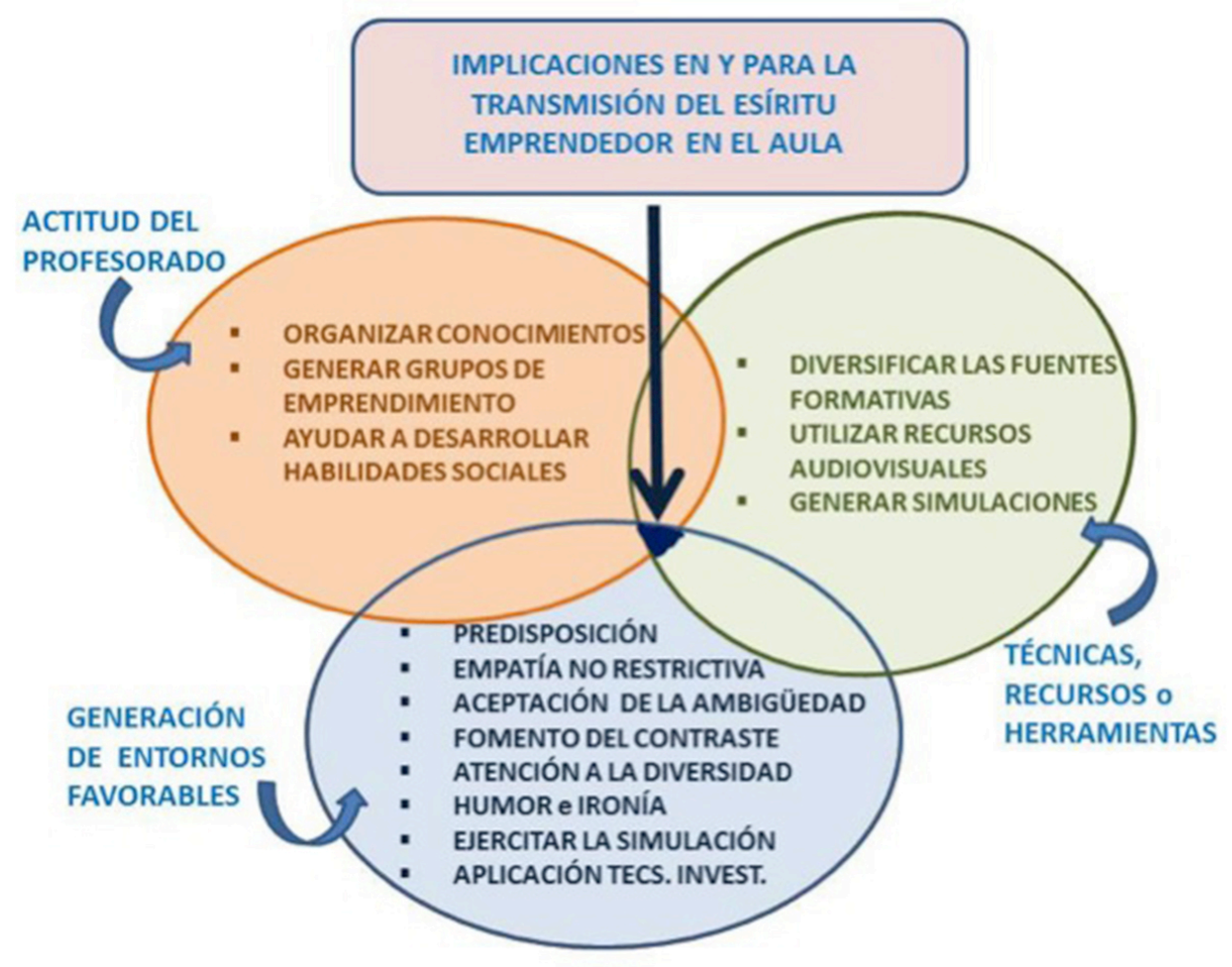

Figura 3.- Modelo y claves facilitadoras para la transmisión del espíritu emprendedor en el aula. Fuente: Elaboración propia.

ISSN: 07 I8-2724. (http://www.jotmi.org) 


\section{Bibliografia}

AKERLOF, G.A. \& Kranton, R.E. (2005). "Identity and the economics of organizations". Journal of Economic Perspectives.Vol. I9, pp. 9-32. doi: I0.1257/0895330053 | 47930.

ALDRICH, H.E. \& Martínez, M.A. (200I).“Many are called but few are chosen: An evolutionary perspective for the study of entrepreneurship". Entrepreneurship Theory \& Practice. Núm. 25 (summer), pp. 4I-56.

BARON, R.A. (2004). "The cognitive perspective: A valuable tool for answering entrepreneurship's basic "why" questions". Journal of Business Venturing. Vol. 19, núm. 2, pp. 221239. doi: $10.1016 / 50883-9026(03) 00008-9$.

BARONET, J. \& Pitcher, P. (1995). Créativité, leadership, stratégique et entrepreneurship: trois facettes d'une meme réalité. Montreal, Québec: Chaire d'entrepreneurship Maclean Hunter. École des Hautes Études Commerciales-HEC.

BIRD, B. (1989). Entrepreneurial Behaviour. Glenview, IL.: Scott, Foresman \& Co.

BOCCONI, S., Kampylis, P.G. \& Punie, Y. (2012). "Innovating Learning: Key Elements for Developing Creative Classrooms in Europe". Joint Research Centre - Institute for Prospective Technological Studies. European Commission. Publications Office of the European Union: Luxembourg. doi: |0.279|/90566.

BORJAS, G. (I 986). “The Self-Employment Experience of Immigrants”. Journal of Human Resources. Vol. 2I, pp. 485-506. doi: 10.2307//45764.

BORJAS, G.J. \& Bronars, S.G. (1989). "Consumer Discrimination and Self-employment". Journal of Political Economy. Vol. 97, pp. 58I-605. doi: 10.1086/261617.

BOYD, R.L. (1990)."Black and Asian self-employment in large metropolitan areas: a comparative analysis". Social Problems. Vol. 37, pp. 258-272. doi:I0.1525/sp. 1990.37.2.03a00090.

BYGRAVE,W.D. \& Hofer, C.W. (1991)."Theorizing about entrepreneurship". Entrepreneurship Theory and Practice.Vol. I6, núm. I, pp. 7-30.

BYGRAVE,W.D. (1989). “The entrepreneurship paradigm (I): A philosophical look at its research methodologies". Entrepreneurship, Theory and Practice.Vol. 14, núm. 2, pp. 7-26.
COMECHE, J.M. (2004)."Factores explicativos de la existencia de emprendedurismo colectivo en las pyme: aplicación a las empresas de la Comunidad Valenciana". Tesis Doctoral. Universitat de València.

CORRALES, J. (199I). La gestión creativa. Madrid: Paraninfo.

CORREA, P. (20I I). Procesos creativos. Polémikus. Ciencias de la Comunicación - Los Libertadores. Universidad Jorge Tadeo Lozano. Colombia. Pp. II-2I.

DAVIDSSON, P. \& Honig, B. (2003). "The role of social and human capital among nascent entrepreneurs". Journal of Business Venturing.Vol. 18, núm. 3, pp. 301-331. doi: 10.1016/ S0883-9026(02)00097-6.

DELMAR, F. \& Davidsson, P. (2000). "Where do they come from? Prevalence and characteristics of nascent entrepreneurs". Entrepreneurship and Regional Development.Vol. I2, Pp. I-23. doi: I0.1080/089856200283063.

DURÁN, C. (2006). "La creatividad como actitud". Revista Ctrl+G, Escuela de Diseño Gráfico de la Universidad del Pacífico. Santiago de Chile.

FALCK, O., Heblich, S. \& Luedemann, E. (20I2). “Identity and entrepreneurship: do school peers shape entrepreneurial intentions?" Small Business Economics. Vol. 39, pp. 39-59. doi: |0.1007/s | | |87-010-9292-5.

FERNÁNDEZ Huerta, J. (1968).“ “Cómo desarrollar la originalidad y la inventiva del alumno durante la escolaridad?". Madrid:Tiempo y Educación. Ed. Compi.

FERNÁNDEZ, M. \& Kim, K. (1998). “Self-Employment Rates of Asian Immigrant Groups: An Analysis of Intra-Group and Inter-Group Differences". International Migration Review. Vol. 32, pp. 654-68I. doi: 10.2307/2547767.

GARDNER, H. (1993). Creating minds. New York: Basic Books.

GARTNER, W. (1988). "Who is an entrepreneur? Is the wrong question".American Journal of Small Business.Vol. 12, núm. 4, pp. II-32.

HERRÁN,A. de la (2008). Didáctica de la creatividad. Madrid: Universidad Autónoma de Madrid.

IBORRA, M.; Menguzzato, M.y Ripollés, M. (1998). “Creación de empresas internacionales: Redes informales y obtención de recursos". Revista Europea de Dirección y Economía de la Empresa.Vol. 7, núm. 3, pp. 147-160. 
IMBERNÓN, F; Silva, P \& Guzmán, C (20II). Competencias en los procesos de enseñanza-aprendizaje virtual y semipresencial. Comunicar, 36, Pp. 107-II4. doi: 10.39/6/C3620II-03-0I.

KAO, J. (1989). Entrepreneurship, Creativity and Organization. Text, cases and readings. Englewood Cliffs, NJ: Prentice Hall.

LAND, E. (1975). "People should want more from life". Forbes. June I, pp. 48-50.

MACPHERSON, D. (1988). "Self-employment and married women”. Economic Letters. Vol. 28, pp. 28I-284. doi: 10.1016/0165-1765(88)90132-2.

MADDISON, J. (I97I). Le rôle de la radio et de la télévision dans l'alphabetisation. Unesco. Paris.

MARÍN, R. (I980). La creatividad. Barcelona: Ceac Editorial.

MOORE, C.F. (1986). "Understanding entrepreneurial Behaviour", in Pearce II, J.A. \& Robinson, R.B. (eds.).Academy of Management Best Papers Proceedings, pp. 66-70. Forty-sixth Annual Meeting of the Academy of Management. Chicago: Academy of Management.

MURGA, M.A. (1983). La radio educativa en la UNED: utilidad y eficacia. Madrid: UNED.

NEALE, M.C. \& Maes, H.H.M. (2002). Methodology for genetic studies of twins and families. Dordrecht, Netherlands: Kluwer Academic Publishers.

NICOLAOU, N. \& Shane, S. (2009). "Can genetic factors influence the likelihood of engaging in entrepreneurial activity?". Journal of Business Venturing. Vol. 24, pp. I-22. doi: 10.1016/j.jbusvent.2007.II.003.

PASTOR, L. \& Xifra, J. (2010). The Radio Dramatization of Educational Contents: A Higher Education Experience. Comunicar, 35, pp. I2I-I29. doi: I0.39|6/C35-20I0-03-05.

REES, H. \& Shah, A. (1986). "An Empirical Analysis of SelfEmployment in the OK". Journal of Applied Econometrics. Vol. I, pp. 95-108. doi: 10.1002/jae.3950010107.

RIPOLLÉS, M. y Menguzzato, M. (2004). “International entrepreneurship: ¿Nuevas empresas internacionales y/o empresas nacidas globales?", en Roig, S. et al. (eds.). El emprendedor innovador y la creación de empresas de I+D+i, pp. 59-69. Valencia: Universitat de València.
RIPOLLÉS, M.; Menguzzato, M. e lborra, M. (2002). "The internationalisation of new ventures. The Spanish case". International Journal of Entrepreneurship and Innovation 2002. Vol. 3, núm.3, pp. | |9|-200. doi: I0.5367/000000002I0I299| 96.

RITSILA, J. \& Tervo, H. (2002). "Effects of Unemployment on New Firm Formation: Micro-level Panel Data Evidence from Finland". Small Business Economics. Vol. 19, pp. 31-40. doi: 10.1023/A:1015734424259.

SANDI, A.M. (1980). "Información de masas y educación". Perspectives I.Vol. X. París.

SARRAMONA, J. (1984). "Educación de adultos por metodología no-formal". Modelos formales y no formales. I Jornadas de Educación de Adultos. Universidad Popular de Zaragoza. Pp. 33-4I.

SCARR, S. (1992). "Developmental theories for 1990s: Development and individual differences". Child Development. Vol.63, pp. I-19. doi: I0. I I I/j. I467-8624.1992.tb0359|.x.

SCHMIDT, J., Soper, J. \& Facca, T. (2012). "Creativity in the entrepreneurship classroom". Journal of Entrepreneurship Education.Vol. I5, pp. I23-131.

SCHMIDT, J., Soper, J. \& Bernaciak, J. (20/3). "Creativity in the entrepreneurship program:A Survey of the directors of award winning programs". Journal of Entrepreneurship Education. Vol. 16, pp. 31-44.

SEIBER, J.E. (1978). "Lecciones de incertidumbre”, en Guilfort, P. et al. Creatividad e Innovación. Buenos Aires: Ed. Paidos.

SHANE, S. \& Venkataraman, S. (2000). "The promise of entrepreneurship as a field of research". Academy of Management Review. Núm. 25, Pp. 217-226. doi: 10.5465/ AMR.2000.27916II.

SIMONTON, D.K. (1999). Origins of genius. Darwinian perspectives on creativity. New York: Oxford University Press.

TORRANCE, E.P. \& Myers, R.E. (1976). La enseñanza creativa. Madrid: Ed. Santillana.

VAN TREUREN, K., Randall, B. \& Fry, C. (20I2). "Teaching Creativity and Innovation in the Classroom".American Society for Engineering Education. 16,T.638, 13746.

WINSLOW, E.K. \& Salomon, G.T. (1987). "Entrepreneurs are more than non-conformists: They are mildly sociopathic". Journal of Creative Behaviour. Vol. 2I, núm. 3, pp. 202-2I3. doi: 10.1002/j.2162-6057.1987.tb00477.x. 
WINSLOW, E.K. \& Salomon, G.T. (1993). "Entrepreneurs architects of innovation, paradigm pioneers and change". Journal of Creative Behaviour.Vol. 27, núm. 2, pp. 75-88. doi: 10.1002/j.2162-6057.1993.tb0|391.x. 